Horizons philosophiques

\title{
Code de déontologie Suicide-Action Montréal Inc.
}

\section{Extraits}

Volume 7, numéro 1, automne 1996

Le dernier pont

URI : https://id.erudit.org/iderudit/801030ar

DOI : https://doi.org/10.7202/801030ar

Aller au sommaire du numéro

Éditeur(s)

Collège Édouard-Montpetit

\section{ISSN}

1181-9227 (imprimé)

1920-2954 (numérique)

Découvrir la revue

Citer ce document

(1996). Code de déontologie Suicide-Action Montréal Inc. Extraits. Horizons philosophiques, 7(1), 77-84. https://doi.org/10.7202/801030ar d'utilisation que vous pouvez consulter en ligne.

https://apropos.erudit.org/fr/usagers/politique-dutilisation/ 


\section{CODE DE DÉONTOLOGIE SUICIDE-ACTION MONTRÉAL INC}

\section{Extraits}

\section{Préambule}

Suicide-Action Montréal Inc. (S.A.M.) croit que l'intervention auprès de toute personne qui fait appel à ses services doit se faire dans le respect de cette personne, de sa capacité de gérer sa vie et de prendre elle-même les décisions qui la concernent. S.A.M. considère également que les personnes qui font appel à ses services se trouvent dans une situation où elles sont incertaines quant aux choix qu'elles doivent faire ou aux actions qu'elles doivent poser pour soulager leur souffrance. S.A.M. veut les aider à faire ce choix dans leur meilleur intérêt, ce qui, pour nous, signifie d'abord rester en vie et améliorer leur qualité de vie.

Ces personnes sont généralement dans un état de crise qui se caractérise par une ambivalence quantaux décisions à prendre. Aussi, comme les personnes qui font appel à nous sont souvent vulnérables, c'est-à-dire dans une situation où leur autonomie est diminuée, notre sentiment de responsabilité à leur égard nous amène à formuler les principes, valeurs et règles d'éthique suivants pour encadrer le travail des intervenants* de S.A.M.

\section{Introduction}

Le Code de déontologie présenté ici s'inspire de valeurs et de normes éthiques couramment incluses et utilisées dans le code de déontologie de différentes disciplines professionnelles. Il a été adapté aux besoins de notre organisme et a pour but d'encadrer toutes les activités cliniques offertes à S.A.M. par les bénévoles, stagiaires, consultants ou employés.

* Parintervenant, on entend les bénévoles et les employé-es qui sont en contact avec les usagers. Afin d'alléger la lecture, nous utiliserons le genre masculin. Veuillez garder à l'esprit la féminisation du genre. 
Ce Code contient quatre principes éthiques et définit les valeurs qui y sont rattachées. Ils serviront de référence pour résoudre les problèmes éthiques, et plus précisément pour décider de la moralité d'un comportement ou d'une attitude.

Les quatre principes retenus sont:

\section{I - Le respect de la dignité de la personne \\ II - L'intervention responsable \\ III - L'intégrité dans les rapports \\ IV - La responsabilité envers la société (...)}

1- Principe du respect de la dignité de la personne

Ce principe est affirmé comme premier et plus important. Adopter ce principe, c'est en même temps reconnaître que toutes les personnes ont une valeur innée en tant qu'êtres humains, ce qui implique :

- que chacune des actions qui sont posées à l'égard d'une personne ou d'un groupe de personnes, ou quiont une influence non négligeable envers ces mêmes personnes, doit être décidée et exécutée avec un intéressement à la personne elle-même et pour elle-même.

- qu'en aucun cas on ne doit utiliser une personne comme un moyen pour atteindre une fin.

L'adoption de ce principe suppose l'adhésion aux valeurs suivantes :

\section{- autonomie et autodétermination}

Chaque personne qui est en pleine possession de ses moyens " a le droit de prendre par elle-même et sans entrave les décisions qui la concernent. Le fait d'intervenir dans le pouvoir

** C'est-à-dire qui peut faire des raisonnements justes en rapport avec des motifs valables, qui a une vision réaliste de sa situation, qui ne cherche pas à se faire souffrir, qui semble agir, malgré ses difficultés présentes, dans une continuité par rapport à ses façons de faire et dans le respect de ses intérêts fondamentaux (engagements, relations avec les autres, façon de concevoir la vie). 
de décision d'une personne en des matières qui la concernent doit être justifié par une évaluation que ses capacités actuelles de décision sont gravement perturbées. Même lorsqu'on évalue qu'une personne a un niveau d'autonomie diminué quant à sa capacité de prendre des décisions, il faut tenter de la faire participer à celles-ci (par exemple, les plans d'intervention) et ainsi favoriser une augmentation de son autonomie.

Ceci implique que chaque action ou intervention entreprise à l'égard d'une personne nécessite son consentement (voir principe II, consentement) si l'on veut respecter les valeurs d'autonomie et d'autodétermination.

\section{- confidentialité}

Tous les renseignements qui ont rapport à une personne et qui sont obtenus soit de cette personne elle-même, de ses proches, de professionnels ou d'un organisme, doivent être gardés avec la plus grande confidentialité. Ainsi, les discussions en rapport avec des informations confidentielles devront se faire dans des locaux appropriés et avec les précautions qui s'imposent. Cette obligation de confidentialité se prolonge même après avoir quitté l'organisme. (...)

Limites à la confidentialité. L'obligation à garder des informations confidentielles cesse :

- lorsqu'un consentement explicite à cet effet est donné

- Lorsque la loi ou un jugement y oblige

- dans certaines situations exceptionnelles (voir principe (I "Consentement»)

- s'il s'agit d'informations comportant des menaces physiques réelles ou éventuelles pouvant entraîner des blessures graves ou la mort d'une autre personne.

\section{- promotion de la personne et de ses intérêts}

Toute intervention doit être faite au profit de la personne à laquelle elle s'adresse et ne pas être subordonnée à des intérêts personnels, organisationnels, scientifiques ou autres. 
On se doit de démontrer un respect pour le jugement informé des usagers de nos services.

Dans le cas des personnes dont l'autonomie se trouve diminuée, il est de la responsabilité de tout intervenant de veiller à établir des sauvegardes"** pour protéger leurs droits. Ces sauvegardes s'appliquent en tenant compte du degré de vulnérabilité et de dépendance de ces personnes, de leur manque d'initiative personnelle et volontaire et/ou de leur incapacité à contrôler leur milieu ou leur vie. Elles seront ajustées en fonction de l'évolution du degré de vulnérabilité et de dépendance de la personne, de sorte que son autonomie ne soit pas compromise indûment. (...)

\section{- justice égale pour tous}

Toute personne qui sollicite des services de S.A.M. a droit de bénéficier d'une qualité et d'une quantité de services égales ou équivalentes, eu égard à ses besoins et à la disponibilité de ceux-ci. Cependant, la présence chez l'usager de comportements qui menacent le bon fonctionnement de l'organisme et/ ou une évaluation clinique qui ne permet pas d'entrevoir de réels bénéfices pour lui, constituent des motifs valables de refus. (...)

\section{II- Principe de l'intervention responsable}

Les intervenants de S.A.M. doiventassumer la responsabilité de leurs interventions face aux usagers, leurs collègues de travail et tous les intervenants impliqués dans la relation d'aide ${ }^{* * *}$ ainsi qu'envers l'organisme (S.A.M.).

Le principe de l'intervention responsable repose d'abord sur le respect par les intervenants du principe de dignité de la personne.

Les sauvegardes sont des mesures ou actions visant à protéger un individu vulnérable. Par exemple, il peut s'agir d'appeler Urgences Santé ou d'établir un contrat.

Établir une relation d'aide, consiste à s'engager dans une séquence d'interactions essentiellement verbales avec l'usager, dans le but de lui faciliter à la fois l'expression, la compréhension et la prise en charge de son vécu. 
Une intervention responsable vise à protéger età promouvoir le bien-être des usagers qui s'adressent à S.A.M. La qualité d'une intervention repose sur la capacité des intervenants d'évaluer adéquatement la situation ou le problème qui se présente à eux et d'agir de façon appropriée. Les intervenants doivent s'assurer de minimiser les dommages et maximiser les bénéfices potentiels qui découlent de leurs actions. De plus, ils doivent prendre la responsabilité de tenter de corriger tout effet nocif survenu à la suite d'une de ces actions. La reconnaissance de ce principe repose sur l'acceptation des valeurs suivantes :

\section{- responsabilité envers les usagers}

L'intervention vise en priorité le bien-être physique et psychologique de l'usager. Ainsi, il va de soi que toute relation avec un usager se fasse au bénéfice de celui-ci et dans le cadre des activités offertes par S.A.M. (...)

Cette responsabilité se limite à l'application de moyens appropriés à la situation, sans entraîner une obligation de réussite. Bien que l'objectif principal de l'intervention soit de préserver la vie et d'amener la personne en crise suicidaire à choisir une solution autre que la mort pour résoudre ses problèmes ou éliminer sa souffrance, les intervenants acceptent sa décision finale, quelle qu'elle soit, et respectent sa liberté de choix (l'urgence 8 constitue une exception; voir consentement ci-dessous).

\section{Limites :}

Consentement Le respect du principe d'autonomie de la personne exige que, pour toute intervention, l'on obtienne un consentement libre et éclairé de la part de l'usager. On considère qu'une demande d'aide comporte un consentement implicite de la part de l'usager au service requis.

Par ailleurs, certaines circonstances (menaces homicidaires, urgence 8 , délire psychotique) obligent à des mesures qui contreviennent à la liberté de l'individu et dispensent de l'obligation d'obtenir un consentement. Dans ces circonstances, l'intervenant doit faire tout en son possible pour prévenir ou 
empêcher les conséquences qui résulteraient du geste posé par l'usager. Ceci peut comprendre :

- maintenirle contact téléphonique avecun usagerjusqu'à ce que son appel soit retracé ou la crise résorbée;

- faire appel aux autorités appropriées (par exemple la police ou Urgences Santé). Dans ces cas, l'obligation de confidentialité cesse. (...)

\section{III - Principe de l'intégrité dans les rapports}

La majeure partie des interventions au plan clinique se font dans un contexte particulier de relation d'aide. Cela suppose alors le développement et le maintien de rapports privilégiés entre les usagers qui font appelà nos services et les intervenants de S.A.M. Ces rapports, ou relations, doivent à tous égards et en tout temps viser à établir le respect et la confiance mutuelle sur laquelle s'élaboreront le dialogue et les échanges en vue de faciliter la résolution du problème.

Pour atteindre cet objectif, on devra veiller à l'observation scrupuleuse des devoirs dictés par un principe de justice, ce qui suppose des rapports fondés sur des valeurs d'honnêteté, d'impartialité et de loyauté.

Cela signifie que les intervenants, quel que soit le contexte où se situe leur action, doivent être honnêtes dans leur conduite à propos des renseignements qu'ils fournissent et quant aux limites de leurs interventions. Ils doivent également être fidèles à leurs engagements et sans parti pris afin d'éviter des conflits d'intérêts. Finalement, ils doivent s'assurer que les usagers puissent toujours donner librement leur assentiment aux interventions proposées ou à la transmission de données qui les concernent directement ou indirectement. (...) 


\section{- impartialité}

L'impartialité suppose que l'on s'assure de se placer dans des conditions permettant de porter un jugement objectif, c'està-dire sans que celui-ci ne soit influencé d'une manière quelconque ou compromis par une situation de conflit d'intérêts, que celui-ci soit réel ou apparent.

Ceci implique que :

Les intervenants ne doivent jamais prendre avantage d'un usager d'un point de vue pécuniaire c'est-à-dire :

- $\quad$ il est interdit de solliciter un usager à titre privé ou de le référer à des amis ou connaissances, à l'encontre de la politique de l'organisme pour les références professionnelles

- il est interdit d'accepter de l'argent ou autres avantages en échange de services rendus

Les intervenants ne doivent pas non plus prendre avantage d'un usager d'un point de vue physique ou émotif, c'est-à-dire :

- il est défendu d'entretenir des activités sexuelles avec les usagers.

De plus :

- en aucune occasion on ne doit inciter quelqu'un de façon pressante et répétée à recourir à des services offerts par S.A.M., même si on juge que ceux-ci lui sont nécessaires. En tout temps, les usagers doivent pouvoir librement consentir aux services offerts.

- par souci d'objectivité clinique, on évite d'intervenir, dans le cadre des activités de SAM, auprès des membres de sa famille et de ses proches. (...)

\section{IV- Principe de responsabilité envers la société}

La responsabilité des divers intervenants qui oeuvrent dans le cadre des activités de S.A.M. n'est pas limitée à la seule 
prestation de services de qualité. Elle déborde largement le cadre de l'organisme pour prendre une dimension sociale dont il nous faut tenir compte à chaque instant. (...)

Aussi est-on en droit de s'attendre à ce que ces personnes agissent dans le respect des structures et institutions sociales, ce qui inclut le respect des lois en vigueur. De même doiventelles contribuer à susciter des changements sociaux qui amélioreront la vie en société et favoriseront la prévention du suicide. (...) 